\title{
PEDOMETER-MEASURED WALKING STEPS AND LEVELS OF PHYSICAL ACTIVITY: A STUDY IN KUALA NERUS, TERENGGANU
}

\author{
NUR AFIFAH MUHAMAD ASRI ${ }^{1}$, ASMA' ALI ${ }^{1 *}$, SITI NUR 'AFIFAH JAAFAR ${ }^{1}$, \\ ABBE MALEYKI MHD JALIL ${ }^{2}$ and KHAIRIL SHAZMIN KAMARUDIN ${ }^{1}$ \\ ${ }^{1}$ Department of Food Science, Faculty of Fisheries and Food Science, \\ Universiti Malaysia Terengganu, 21030 Kuala Nerus, Malaysia \\ ${ }^{2}$ School of Nutrition and Dietetics, Faculty of Health Sciences, \\ Universiti Sultan Zainal Abidin, 21300 Kuala Nerus, Malaysia \\ ${ }^{*}$ E-mail: asma.ali@umt.edu.my
}

Accepted 1 December 2020, Published online 25 December 2020

\begin{abstract}
The Malaysia Ministry of Health introduced the 10,000 Steps Program to embrace physical activity and increase the quality of life among the Malaysian population. Thus, this cross-sectional study aims to determine the walking steps, and level of physical activity among adults in Kuala Nerus, Terengganu. A convenience sample of 90 adults aged 18 to 59 years old had their two-days walking steps assessed using an Omron HJ-320 pedometer. Meanwhile, physical activity was measured using International Physical Activity Questionnaire (IPAQ). The Spearman correlation coefficient is used to assess the relationship between the number of walking steps based on the pedometer, and physical activity level. The result shows that the average median steps/day of the respondents was 5096 (3744). Only $6.7 \%$ of respondents achieved the 10,000 recommended steps. The respondents were generally categorized as low active for their walking steps and physical activity level. There was a significantly low relationship between physical activity and walking $(r=0.214, p=0.043)$. This indicates that the physical activity level among respondents was relatively related by their number of walking steps. In conclusion, a pedometer can be part of an effective tool for measuring daily physical activity. Awareness programs are strongly encouraged to increase the physical activity level among the population.
\end{abstract}

Key words: Walking steps, pedometer, physical activity level, Terengganu, Malaysia

\section{INTRODUCTION}

According to the World Health Organization (WHO), physical activity can be described as any movement of the body by skeletal muscles that necessitates energy expenditure (World Health Organization, 2018) which can be fragmented into two core groupings; exercise and non-exercise physical activity. Exercise encompasses structured and repetitive bodily movements while non-exercise physical activity includes standing, going from, and to school or work, or taking part in household chores or jobs (Odunaiya \& Oguntibeju, 2013). Both can be classified by the level of intensity such as light, moderate, and vigorous. Fast swimming and basketball are part of physical exercise for vigorous

* To whom correspondence should be addressed. activities, but for moderate ones, it involves cycling on the field, sports with catch and throws, and lifting hand weights, sit-ups, and push-ups against the walls for the light activities. For non-exercise physical activity, playing with children at a quick pace and heavy planting are examples of the vigorous degree of intensity. Moderate difficulty involves scaling the stairs, mopping the kitchen, scrubbing the bathtub, and general planting. Lastly, a low level of physical activity involves washing dishes and drying. (Ministry of Health, Labour and Welfare of Japan, 2006; WHO Regional Office for the Western Pacific Region, 2009).

The National Health and Morbidity Survey 2015 recorded moderate physical activity (42.2\%) followed by low physical activity $(31.0 \%)$ and high rates of physical activity $(26.8 \%)$ in Malaysian adults aged 18 years old and above. Malaysian Adult Nutrition Survey (MANS) carried out between 
October 2002 and December 2003 denotes that only $14 \%$ of adults have adequate daily physical activity and about $74 \%$ of the adults spent a majority of their daily time in sedentary activities (Poh et al., 2010). The National Health and Morbidity Survey 2015 reveals that the prevalence of overweight and obesity in Malaysia was $30 \%$ and $17.7 \%$ respectively, and has the highest obesity prevalence in Southeast Asia (IPH, 2015). One of the main factors that lead to obesity is physical inactivity. The World Health Organization reported that physical inactivity is the fourth leading risk factor, causing an estimated 3.2 million deaths globally (World Health Organization, 2009). Based on previous studies, the prevalence of physical inactivity in Terengganu is $46 \%$ which is considered high (Amin et al., 2016).

Kuala Nerus is a state which has undergone rapid changes in the growth of its infrastructure and facilities, primarily due to the presence of two public universities. The expansion of fast-food chains, local restaurants, and cafes, and public transport (including e-hailing services) has been immense. On the one hand, these modernizations have been beneficial to the community, but on the other hand, they will result in high rates of obesity. This phenomenon of obesity is linked to physical inactivity (Friend et al., 2017), less walking, and regular intake of fast food (Goon et al., 2014). Thus, physical inactivity has become an emerging healthrelated issue among adults (WHO, 2009). Many researchers have suggested walking measures as one of the modalities to combat obesity. There is a large volume of published studies describing the role of walking to improve the level of physical inactivity. Hence, the Malaysia Ministry of Health has introduced the 10,000 Steps to align its resolutions with the national's precedence in increasing the physical activity level among the Malaysian population.

Nonetheless, there have been few studies in Terengganu to test these variables. In Terengganu, only a few studies have been conducted on adult walking steps (Leong et al., 2017; Kok et al., 2018; Yusoff et al., 2018). Nevertheless, it has not been studied, in particular in Kuala Nerus, in assessing the steps involved and physical activity together. Therefore, this study aims to determine the relationship between walking steps, and physical activity among adults in Kuala Nerus, Terengganu. This research highlights the connection between walking and adult physical activity. Understanding the connection between walking, and physical activity will help groups like workers and students become aware of the importance of preserving a better quality of life.

\section{MATERIALS AND METHODS}

\section{Research design}

This was a cross-sectional study conducted in the district of Terengganu, which is Kuala Nerus. This study involved a total of 90 adults aged between 18 and 59 years old living in Kuala Nerus. The sample size was calculated at $95 \%$ confidence level, with the proportion of the sedentary population is based on national health morbidity survey $(\mathrm{NHMS})=33.5 \%$ (IPH, 2015), and 0.05 of absolute error by using the formula demonstrated by Cochran (1977). Thus, the minimum sample size derived was 86 . The respondents were recruited through convenience sampling, as the respondents were consecutively selected in order of appearance according to their convenient accessibility. The study was approved by the Human Ethics Board of Committees of Universiti Malaysia Terengganu with reference number: UMT/JKEPM/2017/8.

\section{Research instrument}

This study used a self-administered questionnaire for data collection. The questionnaire consisted of three sections: Section A - sociodemographic, Section B - walking steps counts, and Section C physical activity assessment via IPAQ. For section A, the respondents used the self-reported method to fill in a demographic profile including age, gender, race, religion, marital status, monthly household income, and educational level of respondents. Section B was an assessment of walking steps. Each respondent was given an OMRON HJ-320 pedometer and a daily step log to assess their walking steps for one day on the weekend and one day on a weekday. A pedometer is a device that can be used to empower adults to improve their physical activity since the device can measure the distance travelled on foot by measuring the number of walking steps (Gardner et al., 2011). In several research performed in universities and the community and with patients with diabetes, arthritis, and general practice, the use of pedometers to encourage physical activity has been identified (Mckay et al., 2009). A follow-up session was also held for the review of measures. Before the walking assessment, the respondent must listen to a briefing on how to use the pedometer. The proper place to wear the pedometer on the waistband in line with the midline of the thigh was explained to the respondents before their actual use in the study. Respondents returned with a range of walking steps for one weekend and one weekday. The physical activity level measure based on the daily walking step count on the pedometer is as follows: sedentary (less than 5000 steps), low active $(5,000$ to 7,499 
steps), somewhat active (7,500-9,999 steps), active (10,000-12,499 steps) and highly active (12,500 steps) (Tudor-Locke \& Bassett, 2004).

Section $\mathrm{C}$ was a self-reported questionnaire on physical activity via International Physical Activity Questionnaires (IPAQ), a standardized method used to measure the level of physical activity in a subjective manner, which is available at the IPAQ website (www.ipaq.ki.se). The short-form (IPAQ) was being used instead of long-form as the Short Form (IPAQ) fulfill the needs in providing standardized global surveillance and comparison of physical activity levels. Respondents need to recall their physical activity for the last 7 days. Each respondent needs to recall their vigorous activities, moderate activities, walking, and sitting for the last 7 days. The scores and classification were performed based on the standardized protocol of IPAQ scorings (Craig et al., 2003). After the respondent has completed all of the sections in the questionnaire, the data was entered into IBM SPSS Statistics to determine the average walking step count and physical activity level in terms of median metabolic equivalents (METs) score of the respondents.

\section{Data analysis}

Statistical analysis was conducted using IBM SPSS. For the determination of normality, the Kolmogorov Smirnov test was carried out in data analysis. The mean and standard deviations were used to denote parametric data, while the median is defined for non-parametric data. For walking step counts, the data were analyzed based on median step counts between weekdays and weekends. The nonparametric Spearman correlation coefficient (r) was calculated to determine the relationship between walking steps and meaningful physical activity (IPAQ) at $p<0.05$.

\section{RESULTS AND DISCUSSION}

\section{Socio-demographic characteristics}

All 90 respondents had completed the questionnaire and returned it. Table 1 displays the key characteristics and demographic profiles of the respondents. The result indicates that the majority of respondents are Malay, female, and between 18 and 29 years of age. This may represent the actual population of the Kuala Nerus district, with 20-29year-olds being the largest population (Department Statistics of Malaysia, 2015). Approximately 26.6\% of the household income of the respondents ranged from RM 4000 to RM 4999, followed by $24.4 \%$ for RM 5000 and above, and the remaining respondents had incomes below RM3999. The majority of respondents are pursuing a degree. It is important to distinguish the educational level, as young adults with higher education levels were more physically active than other classes. (Trost et al., 2002).

\section{Walking steps assessment}

Based on Table 1, the total number of walking steps shows that adults in Kuala Nerus are walking more on weekdays than on weekends. This finding reflects those of Leong et al. (2017), who also found that adults in Kuala Terengganu are walking less on weekends than on weekdays. A high count on weekdays was recorded due to a total of $70 \%$ of them reporting that their work schedules were involved. In the current analysis, a total of 5485 (3976) steps were taken by respondents for weekdays. However, based on the cut-off scores (Tudor-Locke \& Bassett, 2004), most of the respondents were rated as low active during working days even though they claim to be active during work. This is because most of them are likely to spend their time sitting in the workplace. The majority of respondents are students who used transport, such as cars and motorcycles, to attend school, reducing the number of walking steps for the respondents. Over the weekends, most of the respondents were likely to have a sedentary lifestyle, with 4270 (3896) walking steps counted. Most of the respondents spent their weekends sitting around and wandering around their home. Although higher steps were reported for weekdays compared to the weekend, both are classified as low-active.

In the meanwhile, the average number of walking steps on the pedometer was 5096 (3744). Based on Tudor-Locke and Bassett (2004), adults in Kuala Nerus were rated as "low active" on average. This is also consistent with the findings of Kok et al. (2018), which found that a group of students in his sample were in a low active group. Another explanation for this outcome may be due to gender. Women are more likely to be active in light and moderate-intensity tasks. According to Chan et al. (2014), physical activity levels among women are generally lower than men. According to the Malaysian Adult Nutrition Survey (MANS), the East Coast population had the lowest prevalence of "ever-exercised" at $18.85 \%$ compared to other regions with a prevalence of more than $30 \%$ (Malaysian Adult Nutrition Survey, 2003). Also, a study by Yusoff et al. (2018) among 95 undergraduate students in Terengganu also reported that most of them were sedentary. Another research conducted by Ying et al. (2017) among students and staff at the University of Malaysia Terengganu (UMT) found that the prevalence of total physical inactivity was $43.7 \%$ among adults over 18 years of age. Evidence indicates that increased inactivity with age could be attributable to poor health and psychosocial factors (Moschny et al., 2011). Ayabe et al. (2008) found that 6752 steps per day 
Table 1. Demographic profile of respondents, walking steps classification, and total number of walking steps $(n=90)$

\begin{tabular}{|c|c|c|c|}
\hline Characteristics & Frequency $(n)$ & $\%$ age $(\%)$ & Median (IQR) \\
\hline \multicolumn{4}{|l|}{ Gender } \\
\hline Female & 48 & 53.3 & \\
\hline Male & 42 & 46.7 & \\
\hline \multicolumn{4}{|l|}{ Race } \\
\hline Malay & 87 & 96.7 & \\
\hline Chinese & 3 & 3.3 & \\
\hline \multicolumn{4}{|l|}{ Age } \\
\hline $18-29$ & 54 & 60.0 & \\
\hline $30-39$ & 26 & 28.9 & \\
\hline 40 and above & 10 & 11.1 & \\
\hline \multicolumn{4}{|l|}{ Religion } \\
\hline Islam & 87 & 96.7 & \\
\hline Buddha & 3 & 3.3 & \\
\hline \multicolumn{4}{|l|}{ Monthly income } \\
\hline Less than RM 1999 & 12 & 12.9 & \\
\hline RM 2000 - RM 2999 & 15 & 16.7 & \\
\hline RM 3000 - RM 3999 & 18 & 20.0 & \\
\hline RM 4000 - RM 4999 & 23 & 25.6 & \\
\hline RM 5000 and above & 22 & 24.4 & \\
\hline \multicolumn{4}{|l|}{ Educational level } \\
\hline SPM or equivalent & 11 & 12.2 & \\
\hline Currently doing diploma & 10 & 11.1 & \\
\hline Currently doing degree & 34 & 37.8 & \\
\hline Diploma holder & 17 & 18.9 & \\
\hline Degree holder & 18 & 19.7 & \\
\hline \multicolumn{4}{|l|}{ Marital Status } \\
\hline Single & 52 & 57.8 & \\
\hline Married & 36 & 40.0 & \\
\hline Divorced/Widow & 2 & 2.2 & \\
\hline \multicolumn{4}{|l|}{ Occupation } \\
\hline Student & 46 & 51.1 & \\
\hline Office worker & 37 & 41.1 & \\
\hline Others & 7 & 7.8 & \\
\hline \multicolumn{4}{|l|}{ Awareness of 10000 steps } \\
\hline Yes & 76 & 84.4 & \\
\hline No & 14 & 15.6 & \\
\hline \multicolumn{4}{|c|}{ Physical activity level (walking steps daily) } \\
\hline Sedentary (less than 5000) & 44 & 48.9 & \\
\hline Low active $(5000-7499)$ & 31 & 34.4 & \\
\hline Somewhat active (7500 - 9999) & 9 & 10.0 & \\
\hline Active $(10,000-12500)$ & 5 & 5.6 & \\
\hline Highly active (12500 and above) & 1 & 1.1 & \\
\hline \multicolumn{4}{|l|}{ Number of walking steps } \\
\hline Weekday & & & 5485 (3976) \\
\hline Weekend & & & 4270 (3896) \\
\hline Average (Weekday + Weekend) & & & 5096 (3744) \\
\hline
\end{tabular}

were recorded by a group of adults in a cardiac rehabilitation program. Although the walking steps recorded were higher than the current study, they were still classified as "low active" also based on the cut-off points of the walking steps. The group of adults undergoing recovery has significantly more steps than this research, as they need to undergo therapy and have a strong desire to improve their health (Ayabe et al., 2008).
In this study, the majority of respondents were said to have a sedentary lifestyle. Table 1 indicates that almost half of the respondents $(48.9 \%)$ had a sedentary lifestyle with less than 5000 walking steps followed by $34.4 \%$ of the respondents were physically low, equal to 5000 to 7499 walking steps. Also, $10 \%$ of respondents were very active, while $5.6 \%$ of respondents were active. Just one respondent was classified as highly active based on walking 
steps. Overall, only $6.7 \%$ of respondents followed the recommended steps for public health, that is 10,000 steps or more. This current study deviates from the study done by Abdullah et al. (2015) which showed almost $17 \%$ of the respondents from 112 university employees in Kuala Lumpur achieved the recommended steps goal for public health. Several researchers have called into question the 10,000 steps a day because 10,000 walking steps are typically not practicable through normal day-to-day routines among average people (Choi et al., 2007). According to Le-Masurier et al. (2003), individuals are more likely to meet existing physical activity guidelines if they achieved at least 10,000 steps per day. However, Le-Masurier et al. (2003) also argue that the completion of 10,000 steps does not warrant compliance with the recommendations recorded in terms of length of time to reap the health benefits of physical activity. Therefore, it is ambitious to have a higher $\%$ age of respondents completing the 10,000 steps a day, and this illustrates the results of this study that the recommendation was achieved by no more than $10 \%$ of respondents. Yet these 10,000 steps can be set as a benchmark and inspire people to do more.

\section{International Physical Activity Questionnaire (IPAQ)}

In this section, the respondents completed a questionnaire about time spent being physically active in the last 7 days. Table 2 shows the distribution of respondents according to their respective IPAQ scores. A majority of the respondents were moderate physical active (48.9\%) followed by low physical activity $(28.9 \%)$ and high physical activity (22.2\%). Table 2 illustrates the average METS per minute score of the respondents. The average METS score was 912, which indicates moderate physical activity. The most probable factor affecting the physical activity level is selfefficacy, which is being aware of the importance of motivation in the adoption and maintenance of health-promoting behaviors (Vancampfort et al., 2015). On weekends, most respondents preferred doing sedentary activities rather than doing physical activity.
However, an increase in median METS scores among certain respondents was due to moderate physical activity such as gardening or cleaning the house on weekends. This finding is consistent with that of Hazizi et al. (2014), who reported that most of the respondents $(47.7 \%$ of males and $66.9 \%$ of females) were found to have a moderate level of physical activity. This study deviates from that by Sebastian et al. (2012), which stated that a high value of METS score was found among 463 Brazilian adults accumulating more than $1500 \mathrm{~min}$ of combined moderate and vigorous physical activity per week, representing $29.45 \%$ of the overall respondents.

\section{Relationship between daily walking steps and physical activity level}

A Spearman correlation test was carried out to determine the relationship between walking steps and the International Physical Activity Questionnaire (IPAQ). Based on Table 3, average walking steps increased along with physical activity level based on METS score, indicating a positive correlation $(r=0.214, p=0.043)$. The relationship is considered a low strength relationship. The outcome was similar to that of a previous study by Leong et al. (2017), which showed $r=0.26$, $p<0.01$, and explained that there was a positive low relationship between walking steps and IPAQ score.

These similarities were due to the circumstance that most people in Kuala Nerus not implementing vigorous physical activity in their daily routines and might be due to the same lifestyles and physical activities performed by the respondents. The results of a study by Jahan and Shenoy (2017) showed that steps taken per day were positively correlated with IPAQ scores $(r=0.56, p<0.001)$. Previous work by Mestek et al. (2008) showed that IPAQ results were firmly associated with steps taken per day. Both of these measure physical activity in a linear fashion. Pedometer-determined data provides adequate information to discriminate between levels of physical activity reported on the IPAQ in the freeliving population, which confirms the claim that pedometers can be applicable in physical activity assessment.

Table 2. Distribution of respondents according to their respective IPAQ score $(n=90)$

\begin{tabular}{lcc}
\hline IPAQ Scores & Frequency & Median (IQR) \\
\cline { 2 - 2 } & $\mathrm{n}(\%)$ & $912(736)$ \\
Average METS per min score & - & \\
IPAQ categorized score & $26(28.9)$ & \\
Low physical activity (0-599) & $44(48.9)$ & \\
Moderate physical activity (600-2999) & $20(22.2)$ & \\
High physical activity (3000 and above) & & \\
\hline
\end{tabular}


Table 3. Correlation between daily walking steps and median METS score

\begin{tabular}{lcc}
\hline \multirow{2}{*}{ Variables } & \multicolumn{2}{c}{ Physical activity (METS score) } \\
\cline { 2 - 3 } & r value & p-value \\
\hline Walking steps (weekday) & 0.118 & 0.267 \\
Walking steps (weekend) & 0.290 & $0.006^{\star}$ \\
Walking steps (average) & 0.214 & $0.043^{\star}$ \\
\hline
\end{tabular}

${ }^{*}$ Correlation is significant at $p<0.05$ (2-tailed).

\section{CONCLUSION}

The current study aimed to assess walking and physical activity among adults in Kuala Nerus, Terengganu. One of the most relevant conclusions to emerge from this study is that their walking steps were generally poor. On average, the level of physical activity is low. The association between walking and physical activity was considered poor. There were a few limitations throughout this study. One of them is the self-reading of the pedometer, which was a limitation of the pedometer assessment itself. Self-reported pedometer-measured physical activity data cannot be considered a gold standard for objectively measured physical activity, since it causes bias because the pedometer does not provide an accurate reading. Besides, the cross-section structure of the analysis limits the results of causation. The respondents were not chosen randomly from the population. Given the limitations of the present research, future studies should suggest a more heterogenous, and randomly selected sample. Despite these limitations, the study indicates that future research is required to establish possible causal mechanisms for the relationship between these walking-step components and physical activity level among respondents. A walking intervention study might be a better approach to relationship determination. A minimum of three days of physical activity monitoring is required since it can provide an adequate estimate of pedometer-determined steps per day (Tudor-Locke and Bassett, 2004). Lastly, to make the results more significant, the study must be conducted in a larger area with a larger population.

\section{ACKNOWLEDGEMENTS}

The authors would like to express their gratitude to all the respondents for their full cooperation.

\section{REFERENCES}

Amin, R.B.M. \& Aziz, A.B.A. 2016. Physical inactivity among adults in a rural village of Kuala Terengganu: Knowledge, attitude, social support, and its associated factors. Malaysian Journal of Public Health Medicine, 16(3): 1922.

Abdullah, M., Saat, N.Z.M., Fauzi, N.F.M., Hui, C.Y. \& Kamaralzaman, S. 2015. Association between walking and cardiovascular risk factors in university employees. Journal of Medical Science, 15(2): 105-109.

Ayabe, M., Aoki, J., Ishii, K., Takayama, K. \& Tanaka, H. 2008. Pedometer accuracy during stair climbing and bench stepping exercises. Journal of Sports Science Medicine, 7(2): 249254.

Chan, Y.Y., Lim, L.K.K., Teh, C.H., Lim, K.H., Hamid, H.A.A., Omar, M.A., Ahmad, N.A. \& Kee, C.C. 2014. Prevalence and factors associated with physical inactivity among Malaysian adults. Southeast Asian Journal Tropical Medicine Public Health, 45(2): 467-480.

Choi, B.C., Pak, A.W., Choi, J.C. \& Choi, C.L.E. 2007. Daily step goal of 10,000 steps: a literature review. Clinical and Investigative Medicine, 30(3): E146-E151.

Cochran, W.G. 1977. Sampling Techniques. 3d Ed. Wiley, New York.

Craig, C.L., Marshall, A.L., Sjöström, M., Bauman, A.E., Booth, M.L., Ainsworth, B.E., Pratt, M., Ekelund, U.L.F., Yngve, A., Sallis, J.F. \& Oja, P. 2003. International physical activity questionnaire: 12-country reliability and validity. Medicine \& Science in Sports \& Exercise, 35(8): 1381-1395.

Department of Statistics Malaysia. 2015. Terengganu at a Glance. Department of Statistics Malaysia, Malaysia. 
Gardner, P.J. \& Campagna, P.D. 2011. Pedometers as measurement tools and motivational devices: new insights for researchers and practitioners. Health Promotion Practise, 12(1): 55-62.

Hazizi, A.S., Zahratul Nur, K., Mohd Nasir, M.T., Zaitun, Y. \& Tabata, I. 2014. Comparison of Physical Activity Prevalence among International Physical Activity Questionnaire (IPAQ), Steps/Day, and Accelerometer in a Sample of Government Employees in Kangar, Perlis, Malaysia. Pertanika Journal of Science Technology, 22(2): 401-417.

Institute for Public Health (IPH) National Health and Morbidity Survey 2015 (NHMS 2015). 2015. Volume II-Non-communicable diseases, risk factors \& other health problems [WWW Document]. URL http://iku.moh.gov.my/images/ I K U/D ocument/REPOR T/nhmsreport 2015vol2.pdf (accessed 6.4.2018)

Jahan, N. \& Shenoy, S., 2017. Relation of pedometer steps count \& self-reported physical activity with health indices in middle aged adults. Diabetes \& Metabolic Syndrome: Clinical Research \& Reviews, 11: S1017-S1023.

Kok, J.L., Asma, A., Khairil Shazmin, K. \& Hayati, M.Y. 2018. A Pedometer-based Intervention with Daily Walking Steps and its Relationship with Nutritional Status among Overweight/ Obese University Students in Kuala Terengganu. International Medical Journal of Malaysia, 17(3): 17-28.

Le-Masurier, G.C., Sidman, C.L. \& Corbin, C.B. 2003. Accumulating 10,000 steps: does this meet current physical activity guidelines. Research Quarterly for Exercise and Sport, 74(4): 389-394.

Leong, R.C., Asma, A., Noor Salihah, K. \& Hayati, M.Y. 2017. Assessing walking steps and its relationship with nutritional status among adults in Kuala Terengganu. Malaysian Applied Biology, 46(3): 113-121.

Malaysian Adults Nutrition Survey. 2003. Ministry of Health Malaysia. pp. 115-118.

McKay, J., Wright, A., Lowry, R., Steele, K., Ryde, G. \& Mutrie, N. 2009. Walking on prescription: the utility of a pedometer pack for increasing physical activity in primary care. Patient Education and Counseling, 76(1): 71-76.

Mestek, M.L., Plaisance, E. \& Grandjean, P. 2008. The relationship between pedometer determined and self-reported physical activity and body composition variables in college-aged men and women. Journal of American College Health, 57: 39-44.
Ministry of Health, Labour, and Welfare of Japan. 2006. Exercise and Physical Activity Guide for Health Promotion 2006, Tokyo [WWW Document]. URL https://www.change4health. gov.hk/en/physical_activity/facts/ classification/index.html (accessed 4.4.2018)

Moschny, A., Platen, P., Klaaßen-Mielke, R., Trampisch, U. \& Hinrichs, T. 2011. Barriers to physical activity in older adults in Germany: a cross-sectional study. International Journal of Behavioral Nutrition and Physical Activity, 8(1): 121-131.

National Health \& Morbidity Survey. 2015. Disability prevalence. Ministry of Health.

National Health \& Morbidity Survey. 2015. Obesity prevalence. Ministry of Health.

Odunaiya, N.A. \& Oguntibeju, O.O. 2013. Physical activity in the Management of Diabetes Mellitus. In: Diabetes Mellitus-Insights and Perspectives. O. Oguntibeju (Ed.). InTech Open, London. p. 193.

Poh, B.K., Safiah, M.Y., Tahir, A., Siti Haslinda, N., Siti Norazlin, N., Norimah, A.K. \& Fatimah, S. 2010. Physical Activity Pattern and Energy Expenditure of Malaysian Adults: Findings from the Malaysian Adult Nutrition Survey (MANS). Malaysian Journal of Nutrition, 16(1): 13-37.

Sebastian, E., Gobbi, S., Chodzko-Zajko, W., Schwingel, A., Papini, C.B., Nakamura, P.M. \& Kokubun, E. 2012. The International Physical Activity Questionnaire-long form overestimates self-reported physical activity of Brazilian adults. Public Health, 126(11): 967-975.

Trost, S.G., Owen, N., Bauman, A.E., Sallis, J.F. \& Brown, W. 2002. Correlates of adults' participation in physical activity: review and update. Medicine \& Science in Sports \& Exercise, 34(12): 1996-2001.

Tudor-Locke, C. \& Bassett, D. 2004. How Many Steps/Day Are Enough? Sports Medicine, 34(1): $1-8$.

Vancampfort, D., Stubbs, B., Ward, P.B., Teasdale, S. \& Rosenbaum, S. 2015. Integrating physical activity as medicine in the care of people with severe mental illness. Australian Journal of Psychiatry, 49(8): 681-682.

WHO Regional Office for the Western Pacific Region. 2009. Pacific Physical Activity Guidelines for Adults: Framework for Accelerating the Communication of Physical Activity Guidelines. Manila: World Health Organization Regional Office for the Western Pacific Region. 
World Health Organization (WHO). 2009. Global health risks: mortality and burden of disease attributable to selected major risks. World Health Organization, Geneva.

World Health Organization (WHO). 2018. Global Strategy on Diet, Physical Activity, and Health. World Health Organization.
Ying, A.S., Khairil, Shazmin K. \& Hayati, M.Y. 2017. Evaluation of dietary intake and quality of life on risk of osteoporosis among adults in Universiti Malaysia Terengganu. Malaysian Applied Biology, 46(3): 123-129.

Yusoff, N.A.M., Ganeson, S., Ismail, K.F., Juahir, H., Shahril, M.R., Lin, L.P. \& Rajikan, R. 2018. Physical activity level among undergraduate students in Terengganu, Malaysia using pedometer. Journal of Fundamental Applied Science, 10(1S): $512-522$. 